\title{
Light Trapping in Solar Cells: Can Periodic Beat Random?
}

\author{
Corsin Battaglia, ${ }^{\dagger, *}$ Ching-Mei Hsu, ${ }^{\ddagger}$ Karin Söderström, ${ }^{\dagger}$ Jordi Escarré, ${ }^{\dagger}$ Franz-Josef Haug, ${ }^{\dagger}$ \\ Mathieu Charrière, ${ }^{\dagger}$ Mathieu Boccard, ${ }^{\dagger}$ Matthieu Despeisse, ${ }^{\dagger}$ Duncan T. L. Alexander, ${ }^{\S}$ Marco Cantoni, ${ }^{\S}$ \\ Yi Cui, ${ }^{\ddagger}$ and Christophe Ballif ${ }^{\dagger}$
}

'Institute of Microengineering (IMT), Photovoltaics and Thin Film Electronics Laboratory, Ecole Polytechnique Fédérale de Lausanne (EPFL), 2000 Neuchâtel, Switzerland, ${ }^{\ddagger}$ Department of Material Science and Engineering, Stanford University, Stanford, California 94305, United States, and ${ }^{\S}$ Interdisciplinary Center for Electron Microscopy (CIME), Ecole Polytechnique Fédérale de Lausanne (EPFL), 1015 Lausanne, Switzerland

$\mathrm{T}$ - meet the requirements for a global terawatt-scale deployment of photovoltaics, further efficiency improvements and cost reductions are necessary. Advanced light-trapping schemes contribute to both objectives simultaneously. By integrating properly engineered photonic nanostructures, sunlight can be trapped within the active absorber layer, thereby enhancing light absorption and thus conversion efficiencies. ${ }^{1-10}$ In addition, increasing absorption also allows one to reduce the absorber layer thickness, which impacts production costs and enables the use of less abundant absorber materials and materials with lower carrier diffusion length.

The question whether random or periodic photonic nanostructures lead to better light trapping in solar cells is currently hotly debated and remains controversial. ${ }^{11-19}$ Here we address this issue in the framework of hydrogenated amorphous silicon (a-Si:H) thinfilm solar cells with absorber layer thicknesses of only $250 \mathrm{~nm}$. These cells rely crucially on advanced light management schemes to achieve high conversion efficiencies as the absorption coefficient of a-Si:H becomes small toward the near-infrared region.

A proven approach that has already been successfully employed to increase light trapping in thin-film silicon solar modules on millions of square meters ${ }^{20}$ is the growth of transparent zinc oxide ( $\mathrm{ZnO}$ ) electrodes with a random pyramidal texture by means of chemical vapor deposition (CVD). ${ }^{21,22}$ This texture has demonstrated outstanding light-trapping capabilities and has been instrumental in achieving the current certified world-record conversion efficiency for singlejunction a-Si:H solar cells. ${ }^{23}$

Here we show that tailored periodic arrays of nanocavities provide excellent light trapping and rival the random pyramidal

\section{ABSTRACT}
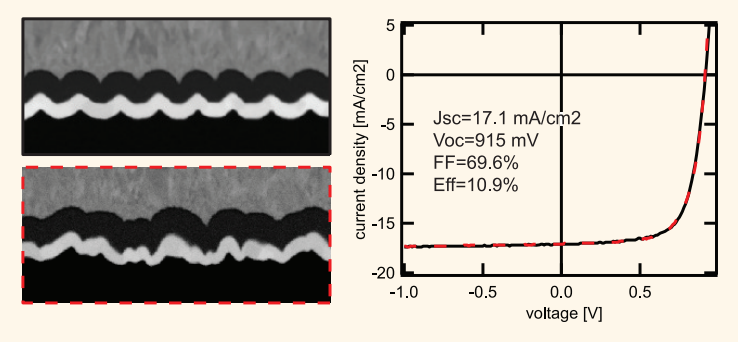

Theory predicts that periodic photonic nanostructures should outperform their random counterparts in trapping light in solar cells. However, the current certified world-record conversion efficiency for amorphous silicon thin-film solar cells, which strongly rely on light trapping, was achieved on the random pyramidal morphology of transparent zinc oxide electrodes. Based on insights from waveguide theory, we develop tailored periodic arrays of nanocavities on glass fabricated by nanosphere lithography, which enable a cell with a remarkable short-circuit current density of $17.1 \mathrm{~mA} / \mathrm{cm}^{2}$ and a high initial efficiency of $10.9 \%$. A direct comparison with a cell deposited on the random pyramidal morphology of state-ofthe-art zinc oxide electrodes, replicated onto glass using nanoimprint lithography, demonstrates unambiguously that periodic structures rival random textures.

KEYWORDS: photovoltaics · solar cells · light trapping · guided modes · Yablonovitch limit · amorphous silicon · nanoimprint lithography · nanosphere lithography

morphology of state-of-the-art $\mathrm{ZnO}$ electrodes. High initial conversion efficiencies of $10.9 \%$ are achieved for both morphologies, allowing us to re-address the question of whether periodic or random structures provide the best light trapping at an unprecedented efficiency level. We examine our results in the framework of waveguide theory, which provides valuable insights into the difference in light-trapping mechanism between periodic and random structures. We identify important light-trapping design principles and indicate potential routes to overcome performance-limiting factors in the quest toward the most efficient light-harvesting scheme.

\section{* Address correspondence to corsin.battaglia@epfl.ch.}

Received for review January 19, 2012 and accepted February 29, 2012.

\section{Published online}

$10.1021 / \mathrm{nn} 300287 \mathrm{j}$

C XXXX American Chemical Society 
a)

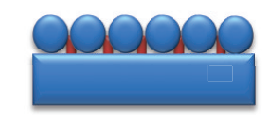

b)

Langmuir-Blodgett

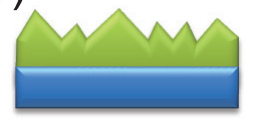

ZnO by chemical

c) vapor deposition

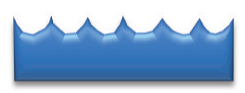

transfer into glass by reactive ion etching

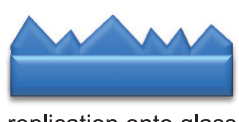

by nanoimprinting $\ln _{2} \mathrm{O}_{3}: \mathrm{H}$ front electrode

by sputtering
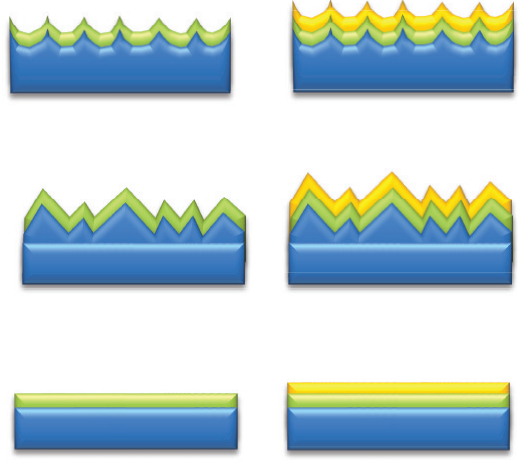

$\mathrm{ZnO}$ back electrode

+ white back reflecto
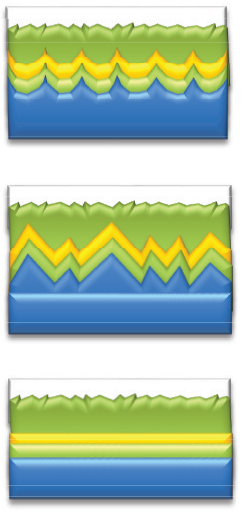

flat glass reference

Figure 1. Schematic process flow of substrate and solar cell fabrication for the periodic nanocavity substrate (a), the random pyramidal texture (b), and the flat reference (c). For details, see text.

Periodic arrays of nanocavities on glass were fabricated using nanosphere lithography (NSL) ${ }^{24}$ (see Figure 1a and Methods section for more details). To allow direct experimental comparison between the periodic nanocavity morphology and the random pyramidal morphology, it is important to isolate the influence of the electrode morphology from the influence of the electrode material properties, for example, the band gap, carrier density, and mobility, which affect the transparency of the material. ${ }^{25,26}$ To deconvolute the effect of morphology on light trapping from the material properties, we used our recently developed highfidelity ultraviolet nanoimprint lithography (UV-NIL) technique $e^{9,27-29}$ to transfer the random pyramidal morphology of state-of-the-art $\mathrm{ZnO}$ electrodes onto a transparent lacquer (see Figure 1b), which behaves optically identically to glass. Details on the deposition of the transparent high-mobility hydrogenated indium oxide $\left(\mathrm{In}_{2} \mathrm{O}_{3}: \mathrm{H}\right)$ front electrodes, the silicon layer stack, and the back contact are given in the Methods section.

\section{RESULTS AND DISCUSSION}

Figure 2a,b presents atomic force microscopy (AFM) images of the periodic nanocavity morphology and the random pyramidal morphology, respectively. For a quantitative characterization of the two morphologies, we extracted height and angle histograms shown in Figure $2 c, d$ from the AFM images. From the height histogram, we see that the nanocavities have a maximum peak-to-valley height of about $150 \mathrm{~nm}$. The randomly sized pyramids have a slightly wider height distribution with maximum heights of up to $300 \mathrm{~nm}$ but an average height similar to the nanocavities. The angle histograms for the two structures are similar, but with the nanocavities exhibiting slightly smaller angles. Figure 2c, d also shows height and angle histograms of suboptimal (over-) smoothed nanocavities, which will be discussed later. Radially averaged autocorrelation functions calculated from the two AFM images are
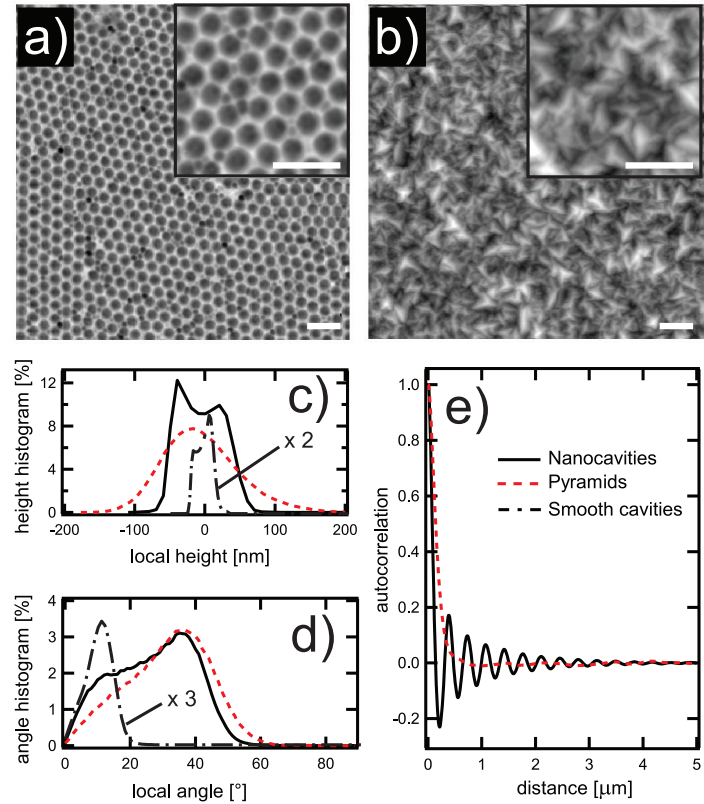

Figure 2. AFM images of the periodic nanocavity array fabricated by NSL (a) and the random pyramidal texture replicated by UV-NIL (b). All scale bars $1 \mu \mathrm{m}$. Local height (c) and angle (d) histograms and radially averaged autocorrelation functions (e) extracted from the AFM images. Bin widths of the histograms are $10 \mathrm{~nm}$ and $1^{\circ}$, respectively.

shown in Figure 2e. The oscillating behavior of the autocorrelation function of the nanocavity array is clear evidence for long-range order with periodicity $a=$ $350 \mathrm{~nm}$, which is also apparent from the AFM image in Figure 2a. Although the nanocavities come in domains, the decay of the oscillation amplitude is sufficiently low to ensure that the coherence length of the periodic nanocavity array extends over several wavelengths of light absorbed by a-Si:H. For the pyramidal morphology, no such oscillations are observed and the system is thus considered to be random. The initially lower decay rate of the autocorrelation function for the pyramidal morphology compared to the 

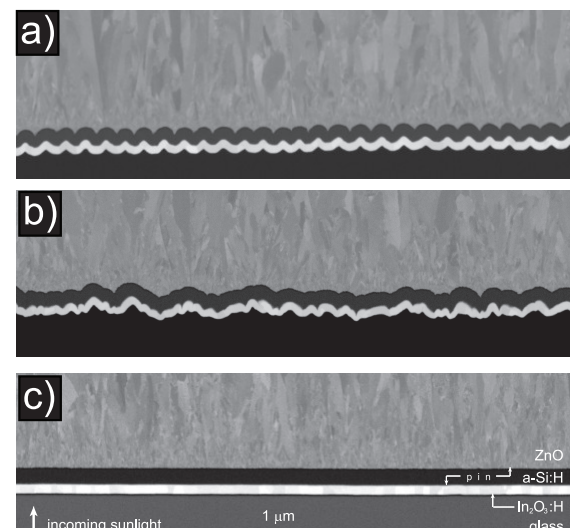

d)

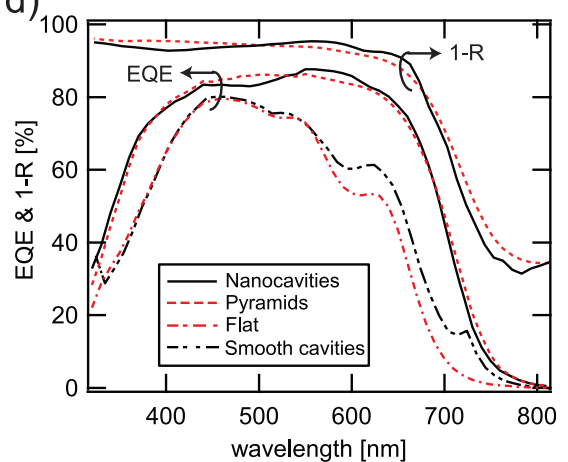

Figure 3. Cross sections of the a-Si:H solar cells fabricated on the periodic nanocavity array (a), the random pyramidal texture (b), and a flat glass reference substrate without light trapping (c), imaged using FIB-SEM. In (c), we also indicate the location of the constituent layers, including the thin $\mathrm{p}$ - and $\mathrm{n}$-type doped silicon layers, which do not exhibit sufficient contrast to be distinguished from the intrinsic (i) silicon layer. Corresponding EQE characteristics are shown in (d). For the cells on the optimized nanocavities and the pyramids, the optical reflectance $R$ is also shown (in the form $1-R)$.

nanocavity morphology is related to the slightly larger feature size.

Figure 3 presents cross sections milled by a focused ion beam (FIB) and imaged by scanning electron microscopy (SEM) across the a-Si:H solar cells fabricated on the optimized periodic nanocavity (a) and random pyramid (b) substrates. A cell on the flat reference substrate without light trapping (c) is also included. Figure $3 \mathrm{~b}$ shows that the pyramidal morphology contains features with a characteristic length scale similar to the nanocavities in Figure 3a. External quantum efficiencies (EQE) measured for these three cells are shown in Figure $3 d$, as is the EQE of a cell on the suboptimal (over-) smoothed nanocavities, which will be discussed later. The effect of light trapping is manifested as a massive enhancement of the EQE in the red part of the spectrum (above $550 \mathrm{~nm}$ ) compared to the flat morphology, but the blue and green part (below $550 \mathrm{~nm}$ ) also benefits strongly from an improved coupling of light into the silicon absorber layer, as the nanotextured interface reduces reflection of light at the glass $-\ln _{2} \mathrm{O}_{3}: \mathrm{H}-$ silicon interfaces. In terms
TABLE 1. Characteristics of a-Si:H Solar Cells Deposited on Periodic Nanocavities, Random Pyramids, and a Flat Reference Substrate

\begin{tabular}{lcccc} 
substrate & $\boldsymbol{J}_{\mathbf{s c}}\left(\mathbf{m A} / \mathbf{c m}^{2}\right)$ & $\boldsymbol{V}_{\mathbf{~ o c}}(\mathbf{m V})$ & $\mathbf{F F}(\%)$ & efficiency (\%) \\
\hline nanocavities & 17.1 & 915 & 69.6 & 10.9 \\
pyramids & 17.1 & 913 & 69.7 & 10.9 \\
flat & 12.0 & 914 & 72.4 & 7.9 \\
\hline
\end{tabular}

of short-circuit current density $\left(J_{\mathrm{sc}}\right)$, a gain of more than $5 \mathrm{~mA} / \mathrm{cm}^{2}$ (more than 40\%) is achieved (see Table 1 for a summary of cell performance characteristics) with the introduction of nanotextured interfaces.

A comparison of the EQE (Figure $3 \mathrm{~d}$ ) of the nanotextured cells reveals that the cavities and pyramids perform almost identically in the blue up to $440 \mathrm{~nm}$ and in the red above $640 \mathrm{~nm}$. From 440 to $540 \mathrm{~nm}$, the EQE for the nanocavities shows a slight dip, which is compensated by an improved spectral response from 540 to $640 \mathrm{~nm}$, resulting in a $J_{\mathrm{sc}}$ of $17.1 \mathrm{~mA} / \mathrm{cm}^{2}$ for both structures (Table 1). This result clearly demonstrates that, with respect to light trapping, periodic structures may perform at least as well as random structures.

We now discuss our results from a theoretical point of view. For a random structure which fully randomizes the incoming light, theory predicts a maximum absorption enhancement of $4 n^{2}$, where $n$ is the refractive index of the absorber material. This is the famous Yablonovitch limit. ${ }^{30}$ Yu et al. ${ }^{31}$ recently showed that, for a periodic triangular grating, the maximum absorption enhancement can be as high as $8 \pi / \sqrt{ } 3 n^{2} \approx 14.5 n^{2}$. We emphasize that such a high enhancement factor is only achieved for a single wavelength equal to $\sqrt{ } 3 / 2$ times the grating period, but that values above the Yablonovitch limit can be achieved over a broader spectral range. It is also important to note that both limits were derived in the weak absorption limit assuming an absorber layer thickness much larger than the wavelength. For thick absorbers, the guided mode spectrum forms a continuum. However, when the absorber thickness is on the order of the wavelength, as in our case, the discrete nature of the modal structure must be taken into account. ${ }^{32,33}$

Figure 4 a presents the guided mode band structure as a function of the parallel component of the wave vector $k_{\|}$for a flat a-Si:H waveguide calculated by determining the complex poles of the Fresnel coefficients ${ }^{34}$ using the experimentally determined values of the complex refractive indices and layer thicknesses. In this diagram, we also show the light lines (or cones in three dimensions) defined by $E=\hbar c / n \cdot k_{\|}$for air, the glass substrate, the transparent conductive oxide (TCO) electrodes (in our case, $\mathrm{In}_{2} \mathrm{O}_{3}: \mathrm{H}$ at the front and $\mathrm{ZnO}$ in the back), as well as a-Si:H. Here $E$ is the energy, $\hbar$ the reduced Planck constant, $c$ the speed of light, and $n$ the refractive index of the respective material. Guided modes of increasing order, labeled by the index $m$, are found to 


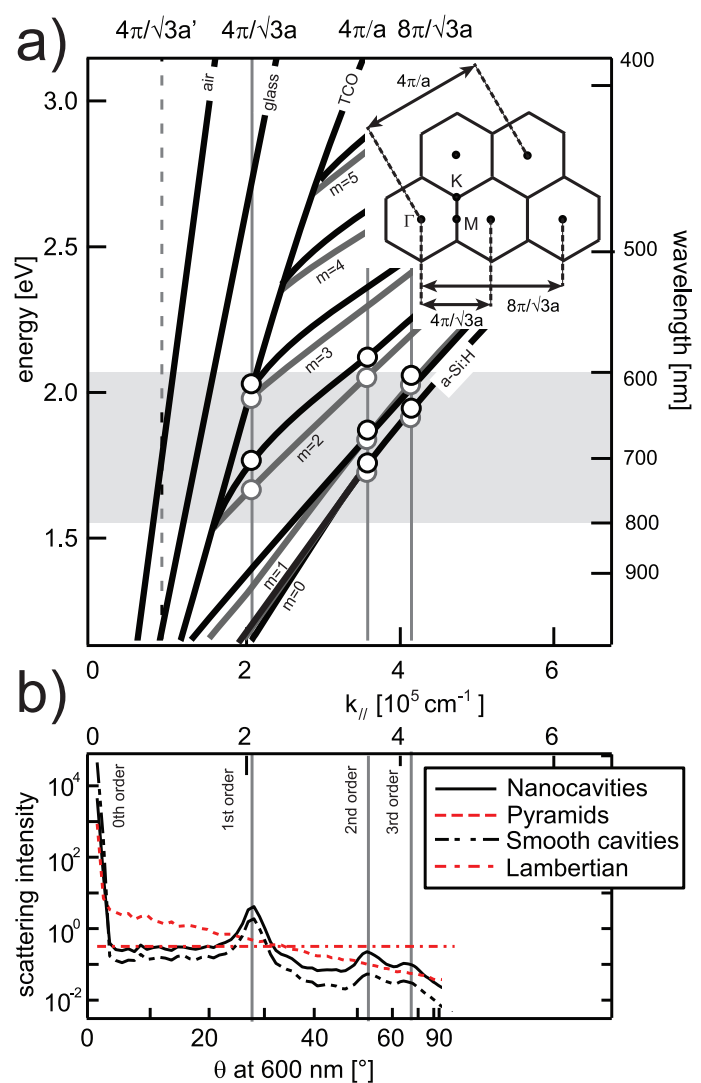

Figure 4. (a) Guided mode band structure for a flat a-Si:H waveguide embedded in TCOs and glass. Guided modes are labeled $m=0, \ldots, 5$. Transversal electric (TE, s-polarized) and transversal magnetic (TM, p-polarized) modes are shown in gray and black lines, respectively. The two $m=0$ modes closely follow the light cone of a-Si:H. The inset sketches the hexagonal Brillouin zones in reciprocal space with high symmetry points and distances between the nearest zone centers indicated. In the band structure, these Brillouin zone centers are represented by thin vertical lines for a periodicity $a=350 \mathrm{~nm}$. For more details, see text. (b) Azimuthally averaged scattering intensity (radiance) as a function of parallel wave vector $k_{\|}$(top scale) and polar scattering angle at a wavelength of $600 \mathrm{~nm}$ (bottom scale).

extend from the light cone of the TCO to the light cone of a-Si:H. Guided modes are also supported by the glass and the TCO, but because of their large thickness, they form a quasi-continuum and are not shown here.

Light incident on a flat waveguide cannot couple to these guided modes. Only radiation modes extending from the energy axis (corresponding to normal incidence) to the light cone of air (representing grazing incidence) can be excited. To couple light into the guided modes, which are characterized by propagation angles exceeding that for total internal reflection, the light must be scattered. As the average height of the nanocavities and pyramids is on the order of or smaller than the absorber layer thickness, we assume for simplicity that the introduction of a texture does not modify the modal structure, which justifies the use of a perturbative point of view.
The difference between periodic and random lighttrapping structures lies in the manner in which they scatter incident light into guided modes. We first discuss the periodic case. The introduction of periodicity divides reciprocal space into Brillouin zones, as shown in the inset of Figure 4a. The first Brillouin zone is centered at $k_{\|}=0$. The centers of higher order Brillouin zones are represented by vertical lines in the band structure in Figure 4a. Due to symmetry requirements, light incident along the normal direction will now be able to excite guided modes (marked by white dots in Figure $4 a$ ) with $k_{\|}$values corresponding to higher order Brillouin zone centers. In fact, light scattered into the second Brillouin zones corresponds directly to the six symmetry-equivalent first-order diffraction peaks of the grating, as can be seen from comparison of Figure $4 a, b$, with the latter showing azimuthally averaged scattering intensities as a function of $k_{\|}$(top scale), which can be related to the wavelength-dependent polar scattering angle $\theta$ in silicon via $k_{\|}=2 \pi n / \lambda \cdot \sin \theta$ (bottom scale). These curves were determined from the AFM images in Figure 2a,b via our Rayleigh-Sommerfeld diffraction algorithm described in ref 35 , as scattering intensities for scattering into silicon cannot be measured experimentally.

For optimum absorption, we have to choose the periodicity in such a way as to maximize the number of excited guided modes in the spectral region of interest. For a-Si:H, this corresponds to the spectral range between 600 and $800 \mathrm{~nm}$ shown in gray in Figure 4a. With the first-order diffraction peak of the $350 \mathrm{~nm}$ grating at $k_{\|}=4 \pi / \sqrt{ } 3 a$, we excite two $m=2$ modes at around $700 \mathrm{~nm}$ as well as the two $m=3$ modes just above $600 \mathrm{~nm}$. Although oversimplified, this model was shown to correctly predict the spectral range where the EQE shows enhancement due to the excited guided mode resonances. ${ }^{36}$ Returning to the experimental EQEs in Figure 3d, we indeed observe a relatively sharp additional resonance at $720 \mathrm{~nm}$ in the EQE of the (over-) smoothed nanocavity array, which is not observed in the EQE of the flat cell. We identify this resonance with the predicted $m=2$ guided modes. A more broad-band enhancement just below $600 \mathrm{~nm}$ is also apparent in the EQE, which we identify with the $m=3$ modes. Note that this guided mode enhancement is superimposed onto an enhancement already present for the flat cell which is due to interference of radiation modes.

To improve the EQE further, more guided modes must be excited. In particular, one should attempt to excite a set of guided modes, allowing a dense coverage of the spectral region of interest. This is achieved with the help of higher diffraction orders. To increase the amount of light that is scattered into higher order diffraction peaks, one can increase the nanocavity height, as can be seen by comparing the scattering intensity curves of the smoothed and optimized nanocavities 
in Figure 4b. For the smooth cavities, $4 \%$ of the total light is scattered out from the zero-order (specular) peak into the first-order diffraction peak. For the optimized nanocavities, we observe a much higher intensity of the first-order diffraction peaks, but the second and third orders exhibit also increased intensity summing to $48 \%$ of the total intensity. Although the intensities of the second- and third-order diffraction peaks are much smaller than that of the first-order peak, their contribution to absorption is believed to be important. Intuitively, in a geometric optical picture, this can be understood by realizing that their propagation angles $\theta$ are much more oblique than for the firstorder peak, which increases the path length in the absorber between successive (lossy) reflections at the (diffractive) silicon-TCO interfaces. In addition, guided modes at higher energy also benefit from a stronger confinement to the absorber layer. Therefore, for the optimized nanocavities, we obtain a much stronger excitation of guided modes by the first-order diffraction peak, but also the excitation of additional guided modes at higher $k_{\|}$values via second- and third-order diffraction. As these additional guide mode resonances allow a much denser coverage of the spectral region of interest, isolated resonances cannot be distinguished anymore and we observe a strong broad-band enhancement in the EQE of the optimized nanocavities in Figure 3d.

Optimum absorption further requires that coupling of the first (and higher) diffraction order(s) to the continuum of radiation modes is avoided in the spectral range of interest. In other words, one has to avoid scattering light into the escape cone (delimited by the light cone of air) to prevent reflection of light out of the cell. This is achieved by selecting a periodicity whose second Brillouin zone center does not intersect the light cone of air in the spectral range of interest. In our case, we chose an even smaller periodicity (resulting in a larger Brillouin zone) to additionally avoid excitation of guided modes which are confined in the glass substrate or the TCOs. In order to guarantee that all excited guided modes are confined in the absorber, we require that the second Brillouin zone center does not cut the light cone of the TCOs in the spectral range of interest. This is the case for the periodicity $a=350 \mathrm{~nm}$ selected for this study. In a geometric optical picture, this condition is equivalent to the requirement that light is scattered into angles sufficiently large that total internal reflection is achieved at the interface between silicon and the TCOs to avoid unnecessary passes through the (parasitically absorbing) TCOs and glass substrate. A slightly smaller Brillouin zone would immediately destroy the confinement to the absorber layer of the $m=3$ modes, whereas a slightly larger Brillouin zone would move the second- and thirdorder diffraction peaks out of the spectral region of interest. Therefore, the selected periodicity of $350 \mathrm{~nm}$ simultaneously guarantees a dense coverage of the spectral region of interest by excited guided modes confined to the absorber layer, while avoiding coupling to lossy radiation or lossy guided modes.

The maximum possible enhancement of $8 \pi / \sqrt{ } 3 n^{2}$ derived by $\mathrm{Yu}$ et al. for the triangular grating is achieved at a periodicity of $2 / \sqrt{ } 3 \lambda .^{31}$ Targeting optimum performance at a wavelength of $\lambda=700 \mathrm{~nm}$, we should therefore select a periodicity of $a^{\prime}=808 \mathrm{~nm}$. From Figure $4 a$, we see that the second Brillouin zone center corresponding to this periodicity, shown as a dashed line in Figure 4a, cuts the air cone much earlier, leading to the excitation of lossy radiation modes below $700 \mathrm{~nm}$. Above $700 \mathrm{~nm}$, first-order diffraction couples light into the guided mode continuum of the glass, which exhibits very low confinement in the absorber. Motivated by this observation, some of us suggested earlier that a lower enhancement limit must apply for absorbers embedded in sufficiently thick dielectric media to support guided modes at these energies. $^{33}$

We now discuss the random case. For periods much larger than the wavelength considered, which asymptotically includes the random case, the upper limit for the enhancement factor derived by $\mathrm{Yu}$ et al. averages to $4 n^{2}$. The Brillouin zones will be very small in this case, allowing the coupling of light to all guided modes but also to the continuum of radiation modes. This can be clearly seen from the scattering profile of the pyramids in Figure 4b, where we do not observe isolated diffraction peaks anymore but a broad continuum extending to $\theta=0$. While for the periodic structure coupling of light into guided modes is only possible at particular energies, the random texture distributes light over all scattering angles and is thus able to couple light at any energy. The integrated (nonspecular) scattered intensity for the random pyramids accounts for $84 \%$ of the total intensity. Although this value is much higher than for the nanocavities, for which we observed only $48 \%$, both textures result in similar EQEs (Figure $3 b$ ). This can be explained by the observation that, in contrast to the nanocavities, the pyramids scatter a considerable amount of light into small angles (i.e., radiation modes). The addition of these radiative loss channels is responsible for the reduction of the limit from $8 \pi / \sqrt{ } 3 n^{2}$ for the triangular periodic case to $4 n^{2}$ for the random case. The $4 n^{2}$ limit is achieved for an ideal random scatterer, such as a Lambertian scatterer, whose scattering profile, which is given by a constant factor $1 / \pi$, is also shown in Figure 4b. Compared to the pyramids, the Lambertian scatterer scatters much more light into large angles, which underlines the importance of large-angle scattering.

A reduction of the maximum achievable absorption enhancement factor for thin-film solar cells must also be expected from the fact that the guided mode spectrum splits into a discrete set of states in a thin 
absorber. Stuart and Hall ${ }^{32}$ observed that a handful of guided modes already lead to a substantial absorption enhancement, which tends asymptotically to the thickfilm limit as the number of modes increases. However, the thick-film absorption limit cannot be achieved with thin absorber layers of only $250 \mathrm{~nm}$.

Furthermore, the theoretical limits were derived under the assumption that each excited mode contributes equally to the total absorption. For the periodic case, this is achieved with an ideal Dirac comb grating, which distributes light equitably over its individual diffraction orders. For the random case, this is fulfilled for the ideal Lambertian scatterer. In experiment, we observe reduced scattering intensity at large angles (Figure 4b). For both periodic and random structures, large-angle scattering can be enhanced by increasing the aspect ratio of the structural features.

Although increasing aspect ratio is beneficial for the optical performance of the cell, this approach is generally detrimental to electrical cell performance because of deviations from layer conformality caused by shadowing during deposition and the formation of morphology-induced areas of porous, low-density material. ${ }^{37,38}$ A trade-off must be found. In our case, this trade-off was achieved by optimizing the morphology of the nanocavities via a hydrofluoric acid etch, while the pyramids were optimized via an argon plasma treatment. Both treatments lead to a smoothening of the morphology, which slightly reduces $J_{\mathrm{sc}}$ but strongly improves the electrical performance, quantified by the open-circuit voltage $\left(V_{\text {oc }}\right)$ and the fill factor (FF) (see Table 1). In particular, within experimental uncertainties, $V_{\text {oc }}$ values as high as on the flat reference cell were achieved on both nanostructured substrates. The reduction of less than $3 \%$ in FF of the cells on the nanostructured substrates compared to the FF of the flat cell can be explained quantitatively by the $40 \%$ increase in current load due to light trapping resulting in increased Ohmic losses in the TCOs. ${ }^{9}$ Although the delicate trade-off between optical and electrical cell performance must be assessed for each morphology individually, it is interesting to note that the performance of the periodic nanocavity and the random pyramid substrate are almost identical such that the current density-voltage $(J(V))$ characteristics in Figure 5 nearly coincide.

It is important to stress that the ultimate performance metric is cell efficiency. Taking this into account, it is all the more remarkable that both morphologies

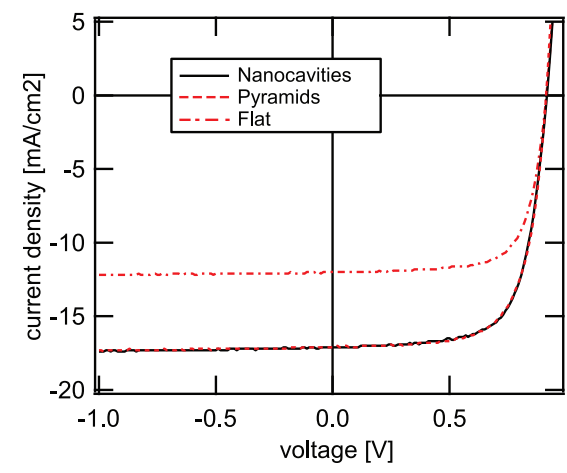

Figure 5. $J(V)$ characteristics for the a-Si:H cells on the optimized periodic nanocavity array, the random pyramidal texture, and the flat reference substrate. The $J(V)$ curves for the cells on the nanocavities and the pyramids are almost identical.

yield initial efficiencies as high as $10.9 \%$. In particular, this is to our knowledge the highest efficiency ever achieved for an a-Si:H solar cell on a periodic substrate. Note that this value may be further improved for both substrates by suppressing the $4 \%$ Fresnel reflection at the flat glass-air interface apparent in Figure $3 \mathrm{~d}$ by applying an antireflective coating. ${ }^{39}$

\section{CONCLUSION}

Although theory predicts that periodic structures should outperform random textures, as they avoid scattering into lossy radiation channels, we showed here at an unprecedented efficiency level of $10.9 \%$ that the performance of state-of-the-art thin-film silicon solar cells is currently limited by the trade-off between optical and electrical performance. One can take advantage of the added optical benefits of periodic structures only if this trade-off can be overcome. Waveguide theory provides an intuitive framework to understand why the chosen period and grating symmetry are efficient. Further efficiency improvements may be achieved by designing scalable morphologies which maximize large-angle scattering via higher aspect ratios but avoid $V_{\text {oc }}$ and FF losses. Recent developments in our lab toward morphology-tolerant silicon layers represent a complementary approach for enhancing light trapping without paying the price of reduced electrical cell performance. ${ }^{40}$ Further work is also required to derive the theoretical upper absorption enhancement limit for realistic thin-film silicon solar cells, which includes the discrete nature of the guided mode spectrum, the influence of (near-field) scattering distribution, and the effect of mode confinement.

\section{METHODS}

Fabrication of Periodic Nanocavity Substrate. Monodisperse silicon oxide spheres with a diameter of $350 \mathrm{~nm}$ were synthesized in a modified Stöber process ${ }^{41}$ and assembled into a hexagonally closed-packed monolayer on a $0.5 \mathrm{~mm}$ thick quartz glass substrate via the Langmuir-Blodgett method. ${ }^{42} \mathrm{~A}$ detailed discussion of the choice for this diameter, which determines the periodicity $a$ of the array, was given before. The sphere diameter was subsequently reduced slightly via reactive ion etching in a trifluoromethane $\left(\mathrm{CHF}_{3}\right)$ /oxygen $\left(\mathrm{O}_{2}\right)$ plasma. 
Aluminum was then evaporated onto the quartz substrate, using the nanospheres as a mask. After subsequent removal of the nanospheres by sonication in ethanol, the aluminum pattern was used as a hard mask for reactive ion etching of nanocavities into the quartz wafer in the $\mathrm{CHF}_{3} / \mathrm{O}_{2}$ plasma. Residual aluminum was removed using an aluminum etching solution, and a buffered hydrofluoric acid etch was used to optimize the surface roughness for cell deposition.

Fabrication of Random Pyramid Substrate. First, a $1.8 \mu \mathrm{m}$ thick $\mathrm{ZnO}$ layer with the characteristic random pyramids was grown by low-pressure CVD on a glass substrate. To optimize the morphology for the growth of the cells, the $\mathrm{ZnO}$ surface was treated for 4 min with an argon plasma. ${ }^{43}$ Then a negative UVNIL stamp was fabricated by nanoimprinting of the master $\mathrm{ZnO}$ texture into a UV-sensitive lacquer, which was spin-coated on a flexible polyethylene naphthalate (PEN) sheet. After curing under UV light and demolding, this stamp was coated with an antisticking layer and used to transfer the positive structure onto a lacquer on a $0.5 \mathrm{~mm}$ thick borosilicate glass substrate. More details including a fidelity analysis of our UV-NIL process can be found in refs 9 and 27-29.

Transparent Front Electrode Deposition. Subsequently, a transparent $\ln _{2} \mathrm{O}_{3}: \mathrm{H}$ electrode ${ }^{25,44}$ with a carrier mobility above $100 \mathrm{~cm}^{2} / \mathrm{N} \cdot \mathrm{s}$, a carrier density on the order of $1 \times 10^{20} \mathrm{~cm}^{-3}$, resulting in a resistivity of about $5 \times 10^{-4} \Omega \cdot \mathrm{cm}$, and a sheet resistance of $45 \Omega / \square$ for a thickness of $110 \mathrm{~nm}$ was sputtered onto both insulating nanostructured substrates as well as a flat glass reference (Figure 1c) and covered by a thin sputtered aluminum-doped $\mathrm{ZnO}$ layer of thickness $20 \mathrm{~nm}$ that served as a protective barrier layer against reduction in the hydrogen-rich plasma during the subsequent silicon layer deposition.

Solar Cell Deposition. Amorphous silicon solar cells were deposited by plasma-enhanced CVD in an industrial reactor with a parallel plate configuration at $200{ }^{\circ} \mathrm{C}$. First, a thin p-type nanocrystalline silicon layer $(5 \mathrm{~nm})$ was deposited to ensure good electrical contact to the $\mathrm{ZnO}$, followed by a $\mathrm{p}$-type amorphous silicon carbide window layer $(10 \mathrm{~nm})$ to boost $V_{\text {oc }}$. Then an intrinsic amorphous silicon absorber layer with a thickness of $250 \mathrm{~nm}$ was deposited followed by a n-type nanocrystalline silicon layer $(2 \times 5 \mathrm{~nm})$ within which a resistive n-type nanocrystalline silicon oxide interlayer $(20 \mathrm{~nm})$ was sandwiched to quench undesired current drains. ${ }^{40}$

Back Electrode Deposition. After deposition of a boron-doped $\mathrm{ZnO}$ back contact, the cells were patterned to $5 \times 5 \mathrm{~mm}^{2}$ by liftoff and sulfur hexafluoride $\left(\mathrm{SF}_{6}\right)$ reactive ion etching. For characterization, a white dielectric back reflector was used, which avoids problems with parasitic plasmonic absorption. ${ }^{45-47}$ All silicon and electrode layers were codeposited to allow for direct comparison.

Solar Cell Characterization. $V_{\text {oc }}$ and FF were calculated from the $J(V)$ characteristics of the cells, measured with a dual-lamp sun simulator in standard test conditions $\left(25^{\circ} \mathrm{C}\right.$, global air mass 1.5 (AM1.5G) spectrum, $1000 \mathrm{~W} \cdot \mathrm{m}^{-2}$ ). $J_{\mathrm{sc}}$ was determined by convolution of the EQE and the incoming photon flux of the AM1.5G spectrum. Optical reflectance $(R)$ measurements on the full cell stacks were carried out on a photospectrometer equipped with an integrating sphere on a larger area of $15 \times 15$ $\mathrm{mm}^{2}$ to improve the signal-to-noise ratio and to minimize the impact of light lost on the sides of the cells.

Substrate Characterization. Substrate morphology was characterized by AFM. Cross sections across the solar cells were milled by FIB and imaged by SEM using a $1.7 \mathrm{kV}$ beam and in-lens backscattered electron detector. For the periodic sample, tomographic methods were used to reconstruct cross-section images precisely aligned to the nanocavity arrays. ${ }^{48}$

Determination of Optical Constants. Complex refractive indices, used for the calculation of the guided mode band structure, were measured by spectroscopic ellipsometry complemented by photothermal deflection spectroscopy for the weakly absorbing regions.

Conflict of Interest: The authors declare no competing financial interest.

Acknowledgment. We thank D. Dominé for stimulating discussions on light scattering, and Z. Holman for careful proofreading and suggestions to improve the manuscript. We acknowledge the help of T. Koida in developing $\ln _{2} \mathrm{O}_{3}: \mathrm{H}, \mathrm{M}$. Leboeuf for assistance in AFM imaging, the Swiss Federal Energy Office and the Swiss National Science Foundation for funding under Project No. 101191 and Grant No. 200021 $12577 / 1$, and the Velux Foundation. Part of this work is supported as part of the Center of Nanostructuring for Efficient Energy Conversion (CNEEC) at Stanford University, an Energy Frontier Research Center funded by the U.S. Department of Energy, Office of Science, Office of Basic Energy Sciences under Award No. DE-SC0001060. A portion of this work is also supported by the U.S. Department of Energy under Award No. DE-FG36-08GOI8004.

\section{REFERENCES AND NOTES}

1. Deckman, H. W.; Wronski, C. R.; Witzke, H.; Yablonovitch, E. Optically Enhanced Amorphous Silicon Solar Cells. Appl. Phys. Lett. 1983, 42, 968.

2. Zhu, J.; Hsu, C.-M.; Yu, Z.; Fan, S.; Cui, Y. Nanodome Solar Cells with Efficient Light Management and Self-Cleaning. Nano Lett. 2010, 10, 1979-1984.

3. Garnett, E.; Yang, P. Light Trapping in Silicon Nanowire Solar Cells. Nano Lett. 2010, 10, 1082-1087.

4. Atwater, H. A.; Polman, A. Plasmonics for Improved Photovoltaic Devices. Nat. Mater. 2010, 9, 205-213.

5. Cao, W.; Myers, J. D.; Zheng, Y.; Hammond, W. T.; Wrzesniewski, E.; Xue, J. Enhancing Light Harvesting in Organic Solar Cells with Pyramidal Rear Reflectors. Appl. Phys. Lett. 2011, 99, 023306.

6. Kuang, Y.; van der Werf, K. H. M.; Houweling, Z. S.; Schropp, R. E. I. Nanorod Solar Cell with an Ultrathin a-Si:H Absorber Layer. Appl. Phys. Lett. 2011, 98, 113111.

7. Ding, I.-K.; Zhu, J.; Cai, W.; Moon, S.-J.; Cai, N.; Wang, P.; Zakeeruddin, S. M.; Grätzel, M.; Brogersma, M. L.; Cui, Y.; et al. Plasmonic Dye-Sensitized Solar Cells. Adv. Energy Mater. 2011, 1, 52-57.

8. Kim, J.; Hong, A. J.; Nah, J.-W.; Shin, B.; Ross, F. M.; Sadana, D. K. Three-Dimensional a-Si:H Solar Cell on Glass Nanocone Arrays Patterned by Self-Assembled Sn Nanospheres. ACS Nano 2012, 6, 265-271.

9. Battaglia, C.; Escarré, J.; Söderström, K.; Erni, L.; Ding, L.; Bugnon, G.; Billet, A.; Boccard, M.; Barraud, L.; Wolf, S. D.; et al. Nanoimprint Lithography for High-Efficiency ThinFilm Silicon Solar Cells. Nano Lett. 2011, 11, 661-665.

10. Battaglia, C.; Escarré, J.; Söderström, K.; Charrière, M.; Haug, F.-J.; Despeisse, M.; Ballif, C. Nanomoulding of Transparent Zinc Oxide Electrodes for Efficient Light Trapping in Solar Cells. Nat. Photonics 2011, 5, 535-538.

11. Sheng, P.; Bloch, A. N.; Stepleman, R. S. WavelengthSelective Absorption Enhancement in Thin-Film Solar Cells. Appl. Phys. Lett. 1983, 43, 579.

12. Eisele, C.; Nebel, C. E.; Stutzmann, M. Periodic Light Coupler Gratings in Amorphous Thin Film Solar Cells. J. Appl. Phys. 2001, 89, 7722.

13. Stiebig, H.; Senoussaoui, N.; Zahren, C.; Haase, C.; Müller, J. Silicon Thin-Film Solar Cells with Rectangular-Shaped Grating Coupler. Prog. Photovoltaics 2006, 14, 13-24.

14. Isabella, O.; Campa, A.; Heijna, M. C. R.; Soppe, W.; van Erven, R.; Franken, R. H.; Borg, H.; Zeman, M. Diffraction Grating for Light Trapping in Thin-Film Silicon Solar Cells. Proceedings of the 23rd European Photovoltaic Solar Energy Conference, Valencia, Spain, 2008; p 2320.

15. Sai, H.; Fujiwara, H.; Kondo, M.; Kanamori, Y. Enhancement of Light Trapping in Thin-Film Hydrogenated Microcrystalline Si Solar Cells Using Back Reflectors with Self-Ordered Dimple Pattern. Appl. Phys. Lett. 2008, 93, 143501.

16. Yu, Z.; Raman, A.; Fan, S. Fundamental Limit of Nanophotonic Light Trapping in Solar Cells. Proc. Natl. Acad. Sci. U.S. A. 2010, 107, 17491-17496.

17. Kelzenberg, M. D.; Boettcher, S. W.; Petykiewicz, J. A.; Turner-Evans, D. B.; Putnam, M. C.; Warren, E. L.; Spurgeon, J. M.; Briggs, R. M.; Lewis, N. S.; Atwater, H. A. Enhanced Absorption and Carrier Collection in Si Wire Arrays for Photovoltaic Applications. Nat. Mater. 2010, 9, 239-244. 
18. Ferry, V. E.; Verschuuren, M. A.; van Lare, M. C.; Schropp, R. E. I.; Atwater, H. A.; Polman, A. Optimized Spatial Correlations for Broadband Light Trapping Nanopatterns in High Efficiency Ultrathin Film a-Si:H Solar Cells. Nano Lett. 2011, 11, 4239-4245.

19. Bhattacharya, J.; Chakravarty, N.; Pattnaik, S.; Slafer, W. D.; Biswas, R.; Dalal, V. L. A Photonic-Plasmonic Structure for Enhancing Light Absorption in Thin Film Solar Cells. Appl. Phys. Lett. 2011, 99, 131114.

20. See, for example, real-time solar panel counter at www. oerlikon.com/solar.

21. Wenas, W. W.; Yamada, A.; Konagai, M.; Takahashi, K. Textured ZnO Thin Films for Solar Cells Grown by Metalorganic Chemical Vapor Deposition. Jpn. J. Appl. Phys. 1991, 30, L441-L443.

22. Fay, S.; Steinhauser, J.; Oliveira, N.; Vallat-Sauvain, E.; Ballif, C. Opto-Electronic Properties of Rough LP-CVD ZnO:B for Use as TCO in Thin-Film Solar Cells. Thin Solid Films 2007, 515, 8558-8561.

23. Benagli, S.; Borrello, D.; Vallat-Sauvain, E.; Meier, J.; Kroll, U.; Hoetzel, H.; Bailat, J.; Steinhauser, J.; Marmelo, M.; Monteduro, G. et al. High-Efficiency Amorphous Silicon Devices on LPCVD-ZnO TCO Prepared in Industrial KAI-M Reactor. Proceedings of the 24th European Photovoltaic Solar Energy Conference, Hamburg, Germany, 2009; 3BO.9.3.

24. Deckman, H. W.; Dunsmuir, J. H. Natural Lithography. Appl. Phys. Lett. 1982, 41, 377.

25. Battaglia, C.; Erni, L.; Escarré, J.; Söderström, K.; Ding, L.; Bugnon, G.; Billetmgrt, A.; Boccard, M.; Barraud, L.; Wolf, S. D.; et al. Micromorph Thin-Film Silicon Solar Cells with Transparent High-Mobility Hydrogenated Indium Oxide Front Electrodes. J. Appl. Phys. 2011, 109, 114501.

26. Battaglia, C.; Escarré, J.; Söderström, K.; Boccard, M.; Ballif, C. Experimental Evaluation of the Light Trapping Potential of Optical Nanostructures for Thin-Film Silicon Solar Cells. Energy Procedia 2012, in press.

27. Escarré, J.; Söderström, K.; Haug, F.-J.; Battaglia, C.; Ballif, C. High-Fidelity Transfer of Nanometric Textures by UV Embossing for Thin Film Solar Cell Applications. Sol. Eneray Mater. Sol. Cells 2011, 95, 881-886.

28. Battaglia, C.; Söderström, K.; Escarré, J.; Haug, F.-J.; Dominé, D.; Cuony, P.; Boccard, M.; Bugnon, G.; Denizot, C.; Despeisse, M.; et al. Efficient Light Management Scheme for Thin Film Silicon Solar Cells via Transparent Random Nanostructures Fabricated by Nanoimprinting. Appl. Phys. Lett. 2010, 96, 213504.

29. Söderström, K.; Escarré, J.; Cubero, O.; Haug, F.-J.; Perregaux, S.; Ballif, C. UV-Nano-Imprint Lithography Technique for the Replication of Back Reflector for n-i-p Thin Film Silicon Solar Cells. Prog. Photovoltaics 2011, 19, 202-210.

30. Yablonovitch, E. Statistical Ray Optics. J. Opt. Soc. Am. 1982, 72, 899.

31. Yu, Z.; Raman, A.; Fan, S. Fundamental Limit of Light Trapping in Grating Structures. Opt. Express 2010, 18, A366-A380.

32. Stuart, H. R.; Hall, D. G. Thermodynamic Limit to Light Trapping in Thin Planar Structures. J. Opt. Soc. Am. A 1997, $14,3001$.

33. Haug, F.-J.; Söderström, K.; Naqavi, A.; Ballif, C. Resonances and Absorption Enhancement in Thin Film Silicon Solar Cells with Periodic Interface Texture. J. Appl. Phys. 2011, $109,084516$.

34. Haug, F.-J.; Söderström, T.; Cubero, O.; Terrazzoni-Daudrix, V.; Ballif, C. Influence of the ZnO Buffer on the Guided Mode Structure in Si/ZnO/Ag Multilayers. J. Appl. Phys. 2009, 106, 044502.

35. Dominé, D.; Haug, F.-J.; Battaglia, C.; Ballif, C. Modelling of Light Scattering from Micro- and Nanotextured Surfaces. J. Appl. Phys. 2010, 107, 044504.

36. Söderström, K.; Haug, F.-J.; Escarré, J.; Cubero, O.; Ballif, C. Photocurrent Increase in n-i-p Thin Film Silicon Solar Cells by Guided Mode Excitation via Grating Coupler. Appl. Phys. Lett. 2010, 96, 213508.
37. Sakai, H.; Yoshida, T.; Hama, T.; Ichikawa, Y. Effects of Surface Morphology of Transparent Electrode on the Open-Circuit Voltage in a-Si:H Solar Cells. Jpn. J. Appl. Phys. 1990, 29, 630.

38. Hsu, C.-M.; Battaglia, C.; Pahud, C.; Ruan, Z.; Haug, F.-J.; Fan, S.; Ballif, C.; Cui, Y. High-Efficiency Amorphous Silicon Solar Cell on Periodic Nanocone Back Reflector. Adv. Energy Mater. 2012, in press.

39. Escarré, J.; Söderström, K.; Despeisse, M.; Nicolay, S.; Battaglia, C.; Bugnon, G.; Ding, L.; Meillaud, F.; Haug, F.-J.; Ballif, C. Geometric Light Trapping for High Efficiency Thin Film Silicon Solar Cells. Sol. Energy Mater. Sol. Cells 2012, 98, 185-190.

40. Despeisse, M.; Battaglia, C.; Boccard, M.; Bugnon, G.; Charrière, M.; Cuony, P.; Hänni, S.; Löfgren, L.; Meillaud, F.; Parascandolo, G.; et al. Optimization of Thin Film Silicon Solar Cells on Highly Textured Substrates. Phys. Status Solidi A 2011, 208, 1863-1868.

41. Bogush, G. H.; Tracy, M. A.; Zukoski, C. F. Preparation of Monodisperse Silica Particles: Control of Size and Mass Fraction. J. Non-Cryst. Solids 1988, 104, 95-106.

42. Hsu, C.-M.; Connor, S. T.; Tang, M. X.; Cui, Y. Wafer-Scale Silicon Nanopillars and Nanocones by Langmuir-Blodgett Assembly and Etching. Appl. Phys. Lett. 2008, 93, 133109.

43. Bailat, J.; Dominé, D.; Schlüchter, R.; Steinhauser, J.; Fay, S.; Freitas, F.; Bucher, C.; Feitknecht, L.; Niquille, X.; Tscharner, T.; et al. High-Efficiency $p$-i-n Microcrystalline and Micromorph Thin Film Silicon Solar Cells Deposited on LPCVD ZnO Coated Glass Substrates. Proceedings of the 4th World Conference on Photovoltaic Energy Conversion, Waikoloa, Hawaii, 2006; p 1533.

44. Koida, T.; Fujiwara, $\mathrm{H}$.; Kondo, M. Hydrogen-Doped $\ln _{2} \mathrm{O}_{3}$ as High-Mobility Transparent Conductive Oxide. Jpn. J. Appl. Phys. 2007, 46, L685-L687.

45. Springer, J.; Poruba, A.; Müllerova, L.; Vanecek, M.; Kluth, O.; Rech, B. Absorption Loss at Nanorough Silver Back Reflector of Thin-Film Silicon Solar Cells. J. Appl. Phys. 2004, 95, 1427.

46. Kim, S.-K.; Ee, H.-S.; Choi, W.; Kwon, S.-H.; Kang, J.-H.; Kim, Y.-H.; Kwon, H.; Park, H.-G. Surface-Plasmon-Induced Light Absorption on a Rough Silver Surface. Appl. Phys. Lett. 2011, 98, 011109.

47. Söderström, K.; Haug, F.-J.; Escarré, J.; Pahud, C.; Biron, R.; Ballif, C. Highly Reflective Nanotextured Sputtered Silver Back Reflector for Flexible High-Efficiency n-i-p Thin-Film Silicon Solar Cells. Sol. Energy Mater. Sol. Cells 2011, 95, 3585-3591.

48. Cantoni, C. G.; Hébert, C.; Knott, G. Large Volume, Isotropic, 3D Imaging of Cell Structure on the Nanometer Scale. Microsc. Anal. 2010, 24, 13. 